\title{
Eva-Maria Krampe
}

\section{Arbeit im Gesundheitswesen: ${ }_{g}$ Reformen ${ }^{\prime \prime}$ auf Kosten der Beschäftigten?}

\section{Gesundheitswesen - ein wachsender Jobmarkt?}

In einer Beilage für Berufsanfänger im Februar diesen Jahres vermeldete die Frankfurter Rundschau Bemerkenswertes über den „Megamarkt" Gesundheitswesen. Bei einem Umsatzzuwachs von heute 220 Milliarden auf 800 Milliarden Euro im Jahr 2030 wurden 200.000 bis 400.000 neue Jobs angekündigt, „die meisten davon im ambulanten und stationären Pflegebereich sowie im zweiten, zumeist privat bezahlten Gesundheitsmarkt". Nur wenige Zeilen weiter konstatierte der Autor zum anderen einen Mangel an Pflegepersonal und demnächst auch an Ärzten, weil junge Menschen angeblich die Belastungen in diesen Berufen scheuen und lieber in den Wellness-Sektor abwandern. Dennoch empfiehlt er die traditionellen Gesundheitsberufe durchaus als zukunftsträchtig, weil sie viele Möglichkeiten, nicht zuletzt auch in den zahlreichen angrenzenden Bereichen und neuen Tätigkeitsfeldern böten (Henniger 2003).

Auch wenn diese Erklärungen etwas oberflächlich daher kommen, beschreiben sie doch den Kern der bereits vollzogenen und zu erwartenden Veränderungen. Man kann den genannten jungen Menschen ebenso wie dem Autor des Artikels ein durch und durch rationales Kalkül bescheinigen. Der immer wieder als gigantisch apostrophierte Gesundheitsmarkt der Zukunft mit unzähligen Chancen für Investoren ebenso wie für innovative junge Menschen ist offensichtlich nicht identisch mit dem, was heute noch von der Mehrheit der Bevölkerung als unser Gesundheitswesen gesehen wird. Vielmehr bezieht sich die Rede vom Zukunftsmarkt weniger auf den traditionellen Teil einer staatlich garantierten umfassenden gesundheitlichen Versorgung als auf den der individualisierten und privat finanzierten Gesunderhaltung. Deshalb war in diesem Beitrag nicht von Sparen oder Kostendämpfung die Rede, sondern nur noch von Expansion. Die aktuellen Berufswünsche und -erwartungen, die in diesem Artikel genannt werden, spiegeln somit besser als die meisten Analysen, wohin der Trend geht. Dass die traditionellen Medizin- und Pflegeberu$\mathrm{fe}$ in einem solchen Kontext an Attraktivität verlieren, erklärt sich schon aus der Tatsache, dass sie mit negativen Stereotypen assoziiert werden. Das hat u.a. damit zu tun, dass die Rahmenbedingungen für die Ausübung dieser Be- 
rufe im Verlaufe der vielfältigen Strukturveränderungen im Gesundheitswesen drastisch verschlechtert wurden - eine Entwicklung die alles andere als zufällig ist. Nimmt man die Ankündigung ernst, dass die Ökonomisierung des Gesundheitswesens unendliche Rationalisierungsreserven mobilisieren soll(te), kann es nicht verwundern, dass eben diese Potenziale in der personalintensiven Gesundheitsbranche insbesondere beim Personal gesucht werden. Ob jedoch das neue Jobangebot im „Megamarkt ${ }^{65}$ Gesundheitswesen weniger belastende, prestige- und einkommensträchtigere Arbeitsmöglichkeiten bietet, darf unter den Bedingungen eines deregulierten Arbeitsmarktes ernsthaft bezweifelt werden.

Die Perspektiven für die "alten" und "neuen" Gesundheitsberufe werden augenfällig, wenn man die Entwicklungen von inzwischen mehr als einem Jahrzehnt Gesundheits(struktur)reformen in den Blick nimmt. Was mit zunächst vorsichtigen Ansätzen zur Einführung ökonomischer Leitlinien und sich dann verschärfenden Budgetierungen begann, hat inzwischen zu einer Welle von (Teil-)Privatisierungen im öffentlichen Gesundheitswesen geführt, mit weitreichenden Folgen nicht nur für die Versicherten und PatientInnen, sondern auch für die Beschäftigten. Gerade an den Umwälzungen hinsichtlich der Struktur und Qualifikation der Beschäftigten und der Art der Beschäftigungsverhältnisse zeigt sich, in welchem Umfang die Ökonomisierung voranschreitet bzw. wie sehr sich die Tendenz zur Privatisierung des Leistungsangebots durchsetzt. Eine Analyse der Umstrukturierungen im Bereich der verschiedenen Beschäftigtengruppen zeigt auch, wie geschickt Konkurrenzen zwischen den einzelnen Berufsgruppen bzw. innerhalb einer Berufsgruppe genutzt werden, um die übergreifende neoliberale Politik durchzusetzen. Die widersprüchlichen Interessen von KrankenhausärztInnen und niedergelassenen ÄrztInnen sowie zwischen Fach- und AllgemeinärztInnen sind zum großen Teil bekannt. Sie haben allerdings noch nicht zu einer ernsthaften Gefährdung des ärztlichen Interessenblocks geführt, obwohl die Berliner Gesundheitspolitik diese Konflikte immer wieder schürt. Anders sieht es bei der Gruppe der beruflich Pflegenden aus; ihre Eliten wollen sich von der Bevormundung durch die Ärzteschaft emanzipieren und setzen dabei auf einen Zuwachs an. Autonomie und Professionalität. Beides erwarten sie von der Ökonomisierung des Gesundheitswesens, welche sie folgerichtig unterstützen. Das ist ein riskantes Manöver, bei dem die bekannten Machtblöcke bisher nicht tangiert wurden, während die Arbeitssituation für die große Mehrheit der Pflegenden ständig prekärer wird.

\section{Unterschiedliche Bedingungen in den Sektoren des Gesundheitssystems}

Diese Prozesse verlaufen in den verschiedenen Bereichen des Gesundheitssystems allerdings durchaus unterschiedlich und mit differierenden Schwerpunkten, je nach der spezifischen Ausgangslage. Das ist zum einen der Tatsache ge- 
schuldet, dass innerhalb des Systems eine Vielzahl von Akteuren und Interessensgruppen auftritt. Auf der Ebene der staatlichen Steuerung sind Bund, Länder und Kommunen involviert; ersterer eher als Steuerungsinstanz, während die beiden letzteren auch als Kostenträger (duale Finanzierung) und Leistungsanbieter (Unikliniken, kommunale Krankenhäuser) auftreten. Als Kostenträger kommen die gesetzlichen und privaten Kranken- und Pflegekassen ins Spiel; als Leistungsanbieter treten neben den öffentlichen Anbietern Verbände der freien Wohlfahrtspflege, die Kirchen und eine kaum überschaubare Menge privater Anbieter, von den niedergelassenen Ärzten und Apothekern über kleine Pflegedienste bis hin zu großen Konzernen oder Klinikketten auf. Ganz zu schweigen von all denen, wie z.B. die pharmazeutische und medizin-technische Industrie, die eher mittelbar auf das Geschehen Einfluss nehmen.

Zum anderen hat die fest etablierte und erstaunlich rigide sektorale Aufgliederung des Gesundheitswesens, nämlich in den stationären und ambulanten Bereich, die Durchsetzung einheitlicher oder auch vereinheitlichender Strategien lange Zeit massiv behindert (vgl. u.a. Wanek 1995, Michelsen 2002). Was sich aus den unterschiedlichen zu Grunde liegenden Strukturen ebenso erklärt wie aus dem Umstand, dass sie in Konkurrenz zueinander auftraten. Dabei muss der stationäre Sektor noch einmal unterschieden werden nach Krankenhäusern der allgemeinen und/oder Akutversorgung, nach Fachkliniken und den Einrichtungen der Rehabilitation, auch stellen die Pflegeheime eine eigene Kategorie im stationären Bereich dar. Der ambulante Sektor stellt ebenso wenig einen einheitlichen Bereich dar, wobei die Konkurrenzen zwischen den niedergelassenen Fachärzten und den Allgemeinmedizinern allgemein bekannt sind (auch Schmacke 2002). Diese Auseinandersetzungen werden nicht nur durch die angestrebte Aufwertung der Allgemein- bzw. Hausärzte erheblich befördert, sondern gewinnen durch die Tatsache, dass die Krankenhäuser immer stärker in der ambulanten Versorgung aktiv werden können, eine zusätzliche Komponente. Dieses wird finanziell gefördert (Stichwort: Integrierte Versorgung) und läuft wohl darauf hinaus, dass die Macht der Kassenärztlichen Vereinigungen, die die Preise aushandeln, ins Wanken gerät. Neben der ärztlichen ambulanten Versorgung existiert der pflegerische ambulante Bereich, der vom ersteren zwar durchaus abhängig, aber dennoch nicht mit ihm verzahnt ist. Akteure mit sektorenübergreifenden Interessen verfolgen andere Strategien als solche, die nur in einem Bereich tätig sind. Das wiederum hängt auch damit zusammen, dass in den jeweiligen Sektoren unterschiedliche Bewertungsund Finanzierungssysteme existieren.

Diese sektoralen Unterschiede, die auch in Bezug auf Abrechnungs- und Bewertungssysteme für Gesundheitsleistungen deutlich werden, stehen den beabsichtigten Rationalisierungen durch integrierte Versorgungskonzepte entgegen. Während die "Preise“ für die ambulante ärztliche Versorgung von den Kassen und den Kassenärztlichen Vereinigungen auf Länderebene ausgehandelt wer- 
den, geht die Abrechnung der Leistungen der Krankenhausärzte in die Pflegesätze und neuerdings Fallpauschalen ein, die die Krankenhäuser mit den Kassen aushandeln, wobei die Ärzte selbst jedoch nach Tarif bezahlt werden. Anders, aber nicht minder komplex sieht es in der pflegerischen Versorgung aus. Während Krankenschwestern/-pfleger im Krankenhaus ebenso wie die Ärzte nach Tarif bezahlt werden und ihre Leistungsberechnung in die Pflegesätze bzw. Fallpauschalen oder neuerdings Diagnosis Related Groups (DRGs) eingeht, gibt es noch die gespaltene Finanzierung pflegerischer Leistungen. Das heißt, dass die Kosten für die Patientenversorgung je nach persönlichem Kontext von bis zu drei verschiedenen Versicherungssystemen übernommen werden. Insbesondere bei chronisch Kranken und Pflegebedürftigen ist es eher die Regel als die Ausnahme, dass die angeordneten Leistungen zum Teil von der Krankenkasse (Behandlungspflege), zum Teil von der Pflegeversicherung (Grundpflege) und zum Teil von der Sozialhilfe übernommen werden. Damit einher geht die Verteilung der politischen Verantwortung auf bis zu drei Ministerien: Gesundheits-, Sozial- und Seniorenministerium. Die Zusammenlegung von Gesundheits- und Sozialministerium nach der letzten Bundestagswahl weist ebenso wie die kürzlich gehörten Plädoyers, Kranken- und Pflegeversicherung zusammenzulegen, darauf hin, dass eine durchaus wünschenswerte Bündelung der Verantwortlichkeit eingeleitet wird. ${ }^{1}$

Schon dieser kursorische Überblick macht deutlich, welche komplexen, vertikal und horizontal stark ausdifferenzierten Strukturen das sektorale System bestimmen, und dass diese durch unterschiedliche Bewertungs- und Finanzierungssysteme nur noch verstärkt werden. Rationalisierungsbestrebungen erscheinen also durchaus vernünftig, wenn es darum geht, das System zu vereinfachen und damit Ausgaben einzusparen, die daraus entstehen, dass die verschiedenen Sektoren nicht kooperieren, sondern geradezu darauf aus sind, dieselbe Leistung mehrfach durchzuführen, d.h. beispielsweise, dass nach einer Krankenhauseinweisung durch einen Facharzt die von ihm vorgenommenen Untersuchungen im Krankenhaus erneut durchgeführt werden (vgl. Bandelow 1998: 53-54, 173-175). Hier eine gewisse Ökonomisierung durchzusetzen, wäre wohl im Interesse auch der Versicherten. Doch die Strategien, die zur Realisierung eingesetzt werden, gehen weit darüber hinaus.

Auf die enorme "Vermachtung" das Systems sei hier nur verwiesen; sie ist ausreichend in der Literatur (Deppe zuletzt 2000; Bandelow 1998; Wanek 1994; Wissmar 1996 u.v.a.m.) beschrieben. Versuche zur Veränderung der vorhandenen Machtstruktur im Kontext der hier beschriebenen Umstrukturierungsprozesse werden im entsprechenden Zusammenhang weiter unten noch thematisiert.

1 Solche Forderungen wurden u.a, von Horst Seehofer, in dessen Amtszeit als Bundesgesundheitsminister die Pflegeversicherung als eigene Versicherung eingeführt wurde, vorgebracht. 


\section{Der Arbeitsmarkt Gesundheitswesen}

In den Jahren 1998 bis 2001 waren im Gesundheitswesen ca. 4,1 Millionen Personen beschäftigt. ${ }^{2}$ Dabei gab es leichte Schwankungen, nämlich eine kurzfristige Abnahme von 1998 bis 2000 (minus 14.000), und einen Anstieg von 35.000 Beschäftigten von 2000 bis 2001 (Statistisches Bundesamt 28.5.2002 bzw. 27.5.2003)。 Berücksichtigt werden bei diesen Zählungen alle Beschäftigten mit Gesundheitsberufen, von den ÄrztInnen und ApothekerInnen über Pflegepersonal, medizinisch-technische und pharmazeutisch-technische AssistentInnen, AltenpflegerInnen und Heilpädagogen bis zu den OptikerInnen und pharmazeutisch kaufmännische Angestellte, und solche, die anderen Berufen im Gesundheitswesen nachgehen. Mit letzteren sind indirekt Beschäftigte, d.h. Beschäftigte in Wirtschaftssektoren, die Vorleistungen für das Gesundheitswesen erbringen, gemeint (GBE 1998: 38). Während die Gesundheitsberufe im Jahr 2000 etwas mehr als 2,6 Millionen Beschäftigte zählten, kamen die anderen Berufe auf knapp über 1,4 Millionen. Dabei zeichnete sich bereits in der Zeit von 1998 bis 2000 der Trend $a b$, dass in den Gesundheitsberufen mehr Personal beschäftigt wurde (plus 65.000 Personen), während in den anderen Berufen die Zahl der Beschäftigten abnahm (minus 77.000 Personen). Von der Beschäftigungszunahme zwischen 2000 und 2001 profitierten erneut die Gesundheitsberufe, die Anzahl der Beschäftigten in anderen Berufen ging abermals zurück. Der Frauenanteil der 4,1 Millionen Beschäftigten im Jahr 2000 lag bei $71 \%$; 68\% der Beschäftigten übten eine Vollzeittätigkeit aus (in der Gesamtwirtschaft lag die Quote bei 78\%), knappe 25\% waren teilzeit(15\% in der Gesamtwirtschaft) und $8 \%$ geringfügig beschäftigt. Der Beschäftigungsrückgang zwischen 1998 und 2000 betraf vor allem Vollzeitbeschäftigte (minus 2,8\%), während die Zahl der Teilzeitbeschäftigten um 5,7\% und die der geringfügig Beschäftigten um 3,3\% anstieg (Statistisches Bundesamt, 28.5.2002, 24.4.2003, eigene Berechnungen). Blickt man allerdings noch einige Jahre weiter zurück, so zeigt sich, dass sich eher qualitative, denn quantitative Veränderungen abzeichnen: Vermerken lässt sich eine Tendenz zum Abbau von Vollzeitbeschäftigungsverhältnissen bei gleichzeitiger Abnahme weniger qualifizierten Personals, während die Gesamtzahl der Beschäftigten über einen längeren Zeitraum eher stabil bleibt. Schon für 1993/1994 nennt der Gesund-

2 In diesem Kontext ist es wichtig zu erwähnen, dass es erst seit 1998 eine differenzierte Gesundheitsberichterstattung gibt, die gemeinsam vom Statistischen Bundesamt und dem Robert Koch Institut durchgeführt wird und die unterschiedlichen Bereiche des Gesundheitswesens analysiert. Zuvor führten neben dem Bundesamt für Statistik und der Bundesanstalt für Arbeit die zahlreichen Kassen und Verbände ihre jeweils eigenen Statistiken, wovon die von der Deutschen Krankenhausgesellschaft veröffentlichten Zahlen für den Krankenhausbereich die zuverlässigsten waren, die allerdings auf diesen Bereich beschränkt blieben.

3 Die Zahlen des Instituts für Arbeitsmarkt- und Berufsforschung für 2001 weichen jedoch davon ab. Dort geht man von einer Zunahme von 30.000 Personen aus, nennt aber als Gesamtzahl 4,5 Millionen Beschäftigte (IAB Materialien 1/2002). 
heitsbericht für Deutschland eine Zahl von 4,2 Millionen Beschäftigten im Gesundheitswesen, wovon 2,2 Millionen Beschäftigte den Gesundheitsberufen und 2 Millionen den anderen Berufen zuzurechen waren (Gesundheitsberichterstattung 1998: 38). Soweit ein erster Überblick, der zeigt, dass die Beschäftigungsentwicklung im Gesundheitswesen durchaus den allgemeinen Entwicklungen entspricht, zumindest was den Abbau von Vollzeitbeschäftigung und die Zunahme von Teilzeitarbeitsplätzen von Frauen im Dienstleistungssektor betrifft (Engelbrech/Jungkunz 2001: 381). Detaillierte Informationen lassen sich erst aus der Betrachtung der einzelnen Sektoren gewinnen.

\subsection{Der Krankenhaussektor}

Die Auswirkungen der strukturellen Reformen des Gesundheitswesens auf die Beschäftigungsverhältnisse von Beginn der 1990er Jahre an müssen vor dem Hintergrund einiger zentraler gesetzlicher Veränderungen betrachtet werden. Dazu gehören die grundlegenden Änderungen in der Krankenhausfinanzierung, die bereits Mitte der 80er Jahre begannen, aber mit der Gesundheitsstrukturreform von 1992 und den dazu gehörenden Nachbesserungen eine neue Stufe erreichten. Budgetierungen, der Rückzug des Bundes aus der Investitionsfinanzierung, das Abrücken vom Selbstkostendeckungsprinzip und die Einführung neuer Berechnungssysteme (Fallpauschalen und Sonderentgelte) veranlassten viele Träger, und zwar Kommunen ebenso wie freigemeinnützige, in den Kliniken Maßnahmen zur Kostensenkung und Erhöhung der Wirtschaftlichkeit einzuführen. In vielen Fällen sah man in der Umwandlung der Krankenhäuser in gemeinnützige $\mathrm{GmbHs}$ oder kommunale Eigenbetriebe eine Maßnahme zur Durchsetzung von wirtschaftlichen Prinzipien. Denn sie beinhaltete neben steuerrechtlichen Veränderungen vor allen Dingen ganz neue Formen der Unternehmensführung und finanziellen Steuerung und ermöglichte damit auch, nach Einhaltung gewisser Schutzfristen, veränderte Arbeitsverträge mit den Beschäftigten. Um dem Gebot der Wirtschaftlichkeit mehr Nachdruck zu verleihen, gingen einige Häuser folgerichtig dazu über, ihre dreiköpfige Klinikleitung, bestehend aus einem Leitenden Arzt, einer Pflegedienstleitung und einem Verwaltungsdirektor, umzuwandeln und die ärztlichen und pflegerischen Leitungen der Verwaltungsdirektion, sprich einem Manager, Ökonomen, Betriebswirt nachzuordnen.

Immer öfter leiten diese Änderungen der Rechtsform und Organisation dann auch Privatisierungen ein. Verschiedentlich wurde das Krankenhausmanagement an private Klinikketten ausgelagert, z.B. in Altenkirchen (RheinlandPfalz), wo das Management von einem der größten deutschen Klinikbetreiber übernommen wurde, obwohl der Landkreis weiterhin einziger Gesellschafter der GmbH blieb. Auch Joint-Ventures nahmen zu. Das heißt, die ursprünglichen Träger verringerten ihre Anteile an den GmbHs zu Gunsten privater 
Konzerne. Inzwischen mehren sich die kompletten Übernahmen durch private Klinikbetreiber. Diese übernahmen in den letzten Jahren zunehmend auch Krankenhäuser der Allgemeinversorgung; selbst Unikliniken stehen vor der Privatisierung. So übernahm die private Helios Kliniken $\mathrm{GmbH}$, die bundesweit 19 Häuser betreibt, im Jahr 2001 das Universitätsklinikum Erlangen (Helios, Pressemitteilung vom 5. April 2001).

\section{Tabelle 1: Allgemeine Krankenhäuser nach Trägern}

\begin{tabular}{|l|c|c|c|c|}
\hline Allgemeine Krankenhäuser & 1994 & 1995 & 2000 & 2001 \\
\hline alle & 2.089 & 2.081 & 2.003 & 1.995 \\
\hline öffentliche & 876 & 863 & 744 & 723 \\
\hline freigemeinnützige & 848 & 845 & 813 & 804 \\
\hline private & 365 & 373 & 446 & 468 \\
\hline
\end{tabular}

Quelle: Krankenhausstatistik - Grunddaten. Statistisches Bundesamt 27.5.2003

Ein Blick auf die Zahlen der 90er Jahre zeigt, welche Verschiebungen hier eintraten. Zunächst ist eine Verringerung der Krankenhäuser insgesamt zu verzeichnen, ihre Zahl sank von 2.411 Häusern im Jahr 1991 auf $2.240 \mathrm{im}$ Jahr 2001. Die Zahl der allgemeinen Krankenhäuser ging von 2.089 im Jahr 1994 auf 1.995 im Jahr 2001 zurück. Gleichzeitig stieg der Anteil der Krankenhäuser in privater Hand (vgl. Tabelle 1) von etwas mehr als 17\% 1994 auf über 24\% 2001. Betrachtet man jedoch die Zahl der Betten in den Krankenhäusern, relativiert sich das Ausmaß der Privatisierungen. Sie betraf hauptsächlich kleinere Einrichtungen, denn nur eine geringe Anzahl, nämlich knappe 8\% der 516.242 Betten in allgemeinen Krankenhäusern stehen in privaten Häusern (Informationssystem GBE 29.5.2003, eigene Berechnungen). Die Privatisierung der Krankenhausversorgung ist also noch begrenzt, zeigt aber eine steigende Tendenz, vor allem auch deshalb, weil sie sich von kleinen, hoch spezialisierten Fachkrankenhäusern hin zu Häusern der allgemeinen Versorgung bewegt. Immer häufiger nämlich verwandeln Städte oder Landkreise ihre Kliniken in Eigenbetriebe (vgl. die Diskussionen in Bremen, Hamburg, Nordfriesland usw. in den letzen zwei Jahren) und leiten damit oft weiter gehende Privatisierungen ein. Auch freigemeinnützige Träger, insbesondere die Kirchen, zeigen eine wachsende Tendenz, ihre Einrichtungen ebenfalls in eine private Rechtsform zu überführen und beginnen so, eigene Klinik- und/oder Heimkonzerne aufzubauen. Ein äußerst erfolgreiches Beispiel dafür ist die Augustinum Gruppe, die Seniorenwohnheime, Kliniken, Schulen und Behinderteneinrichtungen unterhält und im Jahr 2001 einen Umsatzerlös von 249 Millionen Euro erzielte (Augustinum, Eigenwerbung www.augustinum.de 2003).

Wie bereits erwähnt, führt nicht erst die formale Privatisierung oder der Verkauf an eine der Klinikketten zu drastischen Veränderungen in der Art der Unternehmensführung. Schon im Vorfeld werden Maßnahmen durchgeführt, 
die eine Ökonomisierung der Leistungserbringung vorantreiben. Diese umfassen zunächst das Outsourcing von zahlreichen Dienstleistungen, um mit Hauswirtschaft, Küche, Sterilisation, Wäsche nur einige der wesentlichen Bereiche zu nennen. Mit der rechtlichen Umwandlung beginnen aber meistens umfassende organisatorische und ökonomische Restrukturierungen der Klinikbetriebe. Neben dem Outsourcing kann es dann auch zur Zentralisation der genannten Aufgaben kommen, wenn mehrere kleinere Häuser einer Region zu einer GmbH zusammengeführt werden (Ahsen/Grashoff 2003). Bei größeren Häusern werden diese Bereiche oft in Tochterunternehmen ausgegliedert, die der Muttergesellschaft und anderen Auftraggebern die entsprechenden Leistungen wiederum verkaufen. Ein Beispiel dafür ist das ehemalige Städtische Klinikum Kassel, das bereits Mitte der 90er Jahre als vorbildliches Unternehmen auf Seminaren für Krankenhausmanager vorgeführt wurde. Dort wurden sechs voneinander unabhängige Unternehmen gegründet, die auf der einen Seite Kranken-, Alten- und Rehabilitationsdienstleistungen vorhalten und auf der anderen Seite den Küchen- und Reinigungsdienst bzw. die Technikbetreuung anbieten, während es außerdem eine Zentralsterilisation gibt, die ihre Leistungen auch an andere Häuser verkauft.

Die Umwandlung der Rechtsform wird zwar durch einen gewissen, zeitlich begrenzten Bestandsschutz für die Beschäftigten sowohl im Hinblick auf die tarifliche Bezahlung als auch auf den Kündigungsschutz abgefedert. Danach oder für neue MitarbeiterInnen beinhaltet die Aufsplittung eines Unternehmens in mehrere Teilbereiche eine Verschlechterung hinsichtlich der Arbeitsverträge, der Vergütung, der Einhaltung von Mantel-/Tarifverträgen oder der Betriebsratstätigkeit (vgl. dazu ver.di 2002: 23ff). Die Folgen sind aus anderen Wirtschaftssektoren hinreichend bekannt. In welchem Ausmaß diese strukturellen Veränderungen, die oft als eine Besinnung auf die Kernaufgaben apostrophiert werden, vorangetrieben wurden, zeigen die Zahlen in Tabelle 2.

Tabelle 2: Beschäftigte (auf Vollzeitkräfte umgerechnet) in Krankenhäusern

\begin{tabular}{|c|c|c|c|}
\hline Berufsgruppen & 1991 & 2001 & in $\%$ \\
\hline ÄrztInnen & 95.208 & 105.286 & 10,6 \\
\hline Pflegedienst & 326.082 & 373.034 & 14,4 \\
\hline Med.-techn.Dienst & 122.018 & 105.382 & $-13,6$ \\
\hline Funktionsdienst & 78.694 & 78.678 & 0,0 \\
\hline Klin. Hauspersonal & 42.111 & 22.300 & $-47,0$ \\
\hline Wirtschaftsdienst & 95.816 & 74.041 & $-22,7$ \\
\hline Technischer Dienst & 24.549 & 20.473 & $-16,6$ \\
\hline Verwaltungsdienst & 60.704 & 53.905 & $-11,2$ \\
\hline Sonderdienste & 10.906 & 3.332 & $-69,4$ \\
\hline Sonstiges Personal & 19.729 & 11.165 & $-43,4$ \\
\hline
\end{tabular}

Quelle: VdAK/AEV: Ausgewählte Basisdaten des Gesundheitswesens 2002 
Der immense Abbau von Arbeitsplätzen im hauswirtschaftlichen, technischen und Verwaltungsbereich lässt sich wohl nicht allein aus der Schließung einer Reihe von Kliniken ableiten, zumal das direkte Gesundheitspersonal zu- statt abgenommen hat. Außerdem kann man davon ausgehen, dass noch wesentlich mehr Arbeitsplätze verschwunden sind, als diese auf Vollzeitkräfte umgerechneten Zahlen anzeigen, denn gerade in den unterstützenden Bereichen ist der Anteil der Teilzeitkräfte traditionell sehr hoch. Selbst wenn ein Teil dieser ArbeitnehmerInnen einen Arbeitsplatz in einer neu gegründeten Kliniktochter oder einem Reinigungs- oder sonstigem Zulieferbetrieb gefunden hat (Analysen über die Fluktuationen liegen mir nicht vor), so kann man davon ausgehen, dass sie aus dem Tarifgefüge des öffentlichen Dienstes herausgefallen und in den privaten Dienstleistungssektor überführt worden sind, der sich vor allen Dingen durch Niedriglöhne, ungeschützte Arbeitsverhältnisse, geringfügige Beschäftigung und gewerkschaftsfreie Zonen auszeichnet.

Allerdings haben die Strukturveränderungen auch weit reichende Folgen für diejenigen, die weiterhin in den Krankenhäusern beschäftigt sind. $\mathrm{Zu}$ den allgemein bekannten Folgen gehört die Überlastung der KrankenhausärztInnen. Dieser wird in der Entscheidung des Europäischen Gerichtshofs, dass Bereitschaftsdienste als Arbeitszeit anzuerkennen seien, in bestimmter Hinsicht Rechnung getragen. Bislang wurde die Entscheidung in der BRD jedoch noch nicht umgesetzt. Diese Regelungen gelten übrigens auch für die Pflegekräfte. Die Höchstbelastungen für die direkten Gesundheitsberufe werden noch deutlicher, wenn man die sinkende Verweildauer von PatientInnen in den Krankenhäusern (von 12,6 Tagen 1994 auf 9,8 Tage 2001) und das Ansteigen der jährlichen Fallzahlen (von 14,6 Mill. 1994 auf 16,6 Mill. 2001) berücksichtigt (Zahlen Statistisches Bundesamt 28.2.2002). Das heißt, dass viel mehr PatientInnen in wesentlich kürzerer Zeit versorgt werden müssen. Parallel dazu gibt es eine fast gegenläufige Entwicklung: Der Anteil älterer, pflegebedürftiger Menschen, die im Krankenhaus behandelt werden, nimmt zu. Um diese zu betreuen, werden zusätzliche Qualifikationen und auch mehr Zeitaufwand notwendig. Beide Tendenzen zusammen führen zu einer enormen Steigerung der Arbeitsintensität. Dabei zeigen sich die Krankenhäuser kaum bereit, das Personal in angemessenem Maße aufzustocken. Selbst die Urteile des Europäischen Gerichtshofes zur Anerkennung der Bereitschaftsdienste als Arbeitszeit, deren Einhaltung zur Schaffung zahlreicher neuer Arbeitsplätze und zur Entlastung der bereits Beschäftigten führen wird, werden von den Krankenhausgesellschaften aus Kostengründen abgelehnt. Allerdings wollen sie sich um die Umsetzung bemühen, wenn ausreichende finanzielle Mittel zur Verfügung gestellt werden und wenn vor allen Dingen das Arbeitszeitgesetz dahingehend flexibilisiert wird, dass tägliche Höchstarbeitszeiten durch wöchentliche ersetzt werden (Molitor/Walger 2003), womit die Arbeitgeber mehr oder weniger aus dem Schneider wären. 
Um die Kostensenkung der Krankenhausbetriebe weiter zu forcieren, sieht die Gesundheitsgesetzgebung seit langem die konsequente Einführung von Fallpauschalen vor. Damit begann bereits CSU-Gesundheitsminister Horst Seehofer, und die rot-grüne Koalition brachte das Konzept als Diagnosis Related Groups nach australischem Vorbild auf den Weg. Nach anfänglicher Zurückhaltung sind die Krankenhäuser und ihre Verbände mittlerweile fest bei der Umsetzung. Ungefähr die Mälfte aller Häuser hat mit Beginn diesen Jahres auf dieses Berechnungsmodell umgestellt - nicht zuletzt wegen handfester finanzieller Anreize durch die Bundesregierung. Die DRGs ersetzen die Pflegesätze, indem sie ausgehend von der jeweiligen Diagnose und standardisierten Behandlungsplänen die Leistungen der verschiedenen Berufsgruppen im Krankenhaus exakt darstellen sollen und die Kassen auf dieser Grundlage bezahlen. Mit der Einführung soll nicht nur ein enormes Rationalisierungspotenzial aktiviert werden - die Schätzungen über die Einsparmöglichkeiten von Stellen nennen im Bereich der Pflege 18\% bis 20\% weniger Arbeitsplätze (Care konkret 2003). Darüber hinaus beinhaltet diese umfassende Standardisierung ärztlicher und pflegerischer Leistungen, die anschließend auch auf den ambulanten Bereich übertragen werden, einen erheblichen Deprofessionalisierungsschub im Sinne einer Einschränkung beruflicher Handlungsautonomie der beiden Berufsgruppen. Dieser jedoch ist von den Krankenhausträgern, den Kassen und der Politik gewünscht, weil auf diese Weise die bisherige Blockadepolitik der Ärzteschaft ausgebremst werden soll. ${ }^{4}$ Zusätzlich können dann viele Leistungen besser kontrolliert oder auch von billigeren Arbeitskräften erbracht werden, auch der Einsatz von Leiharbeitskräften wird damit wesentlich erleichtert.

Zusammenfassend lässt sich für den Krankenhaussektor vorläufig feststellen, dass die Anfang der 90er Jahre begonnene Ökonomisierung bisher folgende Entwicklungen in Gang gebracht hat:

- die zunehmende Trennung von direkten Gesundheitsdienstleistungen, wie ärztliche und pflegerische Versorgung, und mehr peripheren Leistungen, wie Hauswirtschaft, Ernährung, Technik, Verwaltung;

- damit einhergehend die Verlagerung von Arbeitsplätzen in den privaten Dienstleistungssektor;

- die Zunahme der Arbeitsintensität;

- die Dominanz betriebswirtschaftlicher Prioritäten gegenüber medizinischen und pflegerischen Aspekten;

- die Zunahme von standardisierten Behandlungsabläufen;

- die Einführung von neuen Rechtsformen und Organisationsformen, die die Privatisierung von Einrichtungen ermöglichen und beschleunigen.

4 Vgl. für entsprechende Entwicklungen in den USA: Stone (1996), Morone (1996). 


\subsection{Die Rehabilitation}

Während im Krankenhausbereich Häuser geschlossen und andere häufig in Form einer $\mathrm{GmbH}$ zu einer Einheit mit gemeinsamer Verwaltung und zentralisierten Dienstleistungen zusammengeschlossen wurden, verzeichnet der Rehabilitationssektor nach einer kurzfristigen Stagnation Mitte der 90er Jahre eine Zunahme der Betriebe. Von 1991 bis zum Jahr 2000 stieg die Anzahl der Einrichtungen von 1.180 auf 1.393 und die Zahl der Beschäftigten auf Vollzeitkräfte hochgerechnet von 78.000 auf 120.000 im Jahr 2001, ca. 30\% der Beschäftigten waren Teilzeitkräfte (Norddeutsche Landesbank 2002; Statistisches Bundesamt, 24.4.2003). Die wachsenden Beschäftigtenzahlen beinhalten auch Zuwächse bei hauswirtschaftlichem und technischem Personal und nicht nur bei den ÄrztInnen, Therapeutinnen und Pflegekräften.

Im Gegensatz zum Krankenhaussektor ist die Trägerstruktur bei den RehaEinrichtungen wesentlich stärker von privaten Unternehmen geprägt, wenn auch mit leicht fallender Tendenz. Waren 1995 noch 62\% der Einrichtungen privat, so gab es 2000 nur noch 58\% in privater Hand. Der Anteil der öffentlichen Institutionen blieb im selben Zeitraum stabil, während freigemeinnützige Einrichtungen von knapp 23\% auf 26,6\% zunahmen (Norddeutsche Landesbank 2002: 30). Allerdings verfügen die privaten Unternehmen nicht nur über eine wesentlich höhere Bettenzahl, sondern behandeln auch erheblich mehr Fälle. Auf die jährlich behandelten Fälle umgerechnet, macht der Anteil der Privaten knapp zwei Drittel aus, während Freigemeinnützige und Öffentliche zusammen nur ein gutes Drittel der Fälle behandeln.

Dem Rehabilitationsbereich werden ebenso wie der ambulanten Versorgung positive Wachstumsprognosen gestellt. Hoffnungen auf eine Ausweitung dieses Sektors nähren sich zum einen aus der Tatsache, dass die Krankenhausbehandlungen immer mehr verkürzt und somit Anschlussheilbehandlungen zunehmen werden. Auf der anderen Seite lassen sich gerade in diesem Sektor Angebote der klassischen kurativen Gesundheitsversorgung, die von den Sozialleistungsträgern bezahlt werden, mit darüber hinausgehenden Wellness- und Fitnessangeboten verbinden, die dem zweiten, privaten Gesundheitsmarkt zuzurechnen sind (Norddeutsche Landesbank 2002). Die Anschlussfähigkeit zu beiden Seiten des immer stärker auseinanderfallenden Gesundheitssystems gewährleistet eine gute Marktposition, die die großen Klinkbetreiber längst für sich nutzen, denn nicht wenige von ihnen haben ihren Ursprung im RehaBereich und gehen jetzt dazu über, auch Klinken der Allgemeinversorgung zu übernehmen. Damit verbessern sie ihre Möglichkeiten der Steuerung von Kundenströmen erheblich. Gleichzeitig ergeben sich durch die Vermischung von Klinik- und Hotelleistungen innerhalb einer Einrichtung und/oder eines Konzerns nicht nur Wettbewerbsvorteile, sondern noch ganz andere Möglichkeiten der Tarifgestaltung und der Rekrutierung von Personal, vor allen Din- 
gen in Hinblick auf diejenigen, die nicht den direkten Gesundheitsfachberufen zuzurechnen sind.

\subsection{Die Pflegeheime}

Im Bereich der Pflegeheime, in den nicht nur alte Menschen, sondern auch jüngere nicht mehr zu rehabilitierende Menschen mit schweren Krankheiten oder Behinderungen untergebracht werden, lässt sich eine wesentlich andere Ausgangsituation feststellen. Dafür gibt es zwei Gründe: Während Krankenhaus- und Rehabilitationsaufenthalte von den Krankenkassen bzw. von den Sozialversicherungsträgern bezahlt werden, muss der Aufenthalt in einem Pflegeheim privat finanziert werden. Das heißst, dass nur die Versorgung im Rahmen der Pflegeversicherung je nach Pflegebedürftigkeit und die medizinische Betreuung im Krankheitsfall von den zuständigen Kassen übernommen werden. Die Unterkunft und allgemeine Versorgung müssen die Pflegebedürftigen selbst, gegebenenfalls die Sozialhilfe, zahlen. Außerdem sieht die Trägerstruktur erheblich anders aus als bei den anderen stationären Einrichtungen. Von den 8.500 Heimen im Jahr 2000 haben 57\% einen freigemeinnützigen Träger, u.a. Caritas und Diakonie. Weitere $35 \%$ waren in privater Hand; mehr als ein Viertel der Einrichtungen bietet inzwischen verschiedene Stufen, vom Seniorenwohnen über das betreute Wohnen bis hin zur vollkommenen Pflege an (Adolph/Heinemann 2003; Norddeutsche Landesbank 2002).

Allein aus der Tatsache, dass die Heimunterbringung privat bezahlt werden muss, erklären sich die gravierenden Unterschiede im Angebot. Von der luxuriösesten Ausstattung mit hervorragender Betreuung bis zur Satt-SauberTrocken-Versorgung reicht das Spektrum. Damit ist die Altenversorgung eines der prägnantesten Beispiele dafür, was im Gesundheitswesen geschehen wird, wenn nur noch sog. Grundleistungen über das gesetzliche Versicherungssystem abgedeckt werden und alles andere privat finanziert werden muss. Die Pflegeskandale, die in den letzten Jahren bekannt wurden und die auf qualitativ schlechte, wenn nicht gar unprofessionelle Pflege zurückzuführen waren, gab es nicht allein in privaten Heimen, sondern auch in kommunalen bzw. solchen mit freigemeinnützigen Trägern.

Dementsprechend sieht die Struktur der Beschäftigten in diesem Sektor aus. Von 441.000 Personen, die Ende 1999 in Pflegeheimen tätig waren, waren $84 \%$ Frauen, hatten $21 \%$ eine wie auch immer gestaltete Altenpflegeausbildung, und 10\% eine Krankenpflegeausbildung. Von allen Beschäftigten arbeiteten $48 \%$ auf Vollzeit- und $45 \%$ auf Teilzeitarbeitsplätzen. Die übrigen $7 \%$ waren PraktikantInnen, Auszubildende oder Zivildienstleistende (Statistisches Bundesamt 2001: 17). Besonders dramatisch ist diese Beschäftigtenstruktur angesichts der Tatsache, dass die PatientInnen in den Pflegeheimen heute eine wesentlich intensivere Betreuung benötigen als noch vor wenigen Jahren. 
Denn auf Grund der ausgeweiteten ambulanten Betreuung kommen sie erst dann ins Heim, wenn jede andere Versorgung ausgeschlossen ist. Allenthalben werden die Zustände beklagt, die Überlastung des Personals und dessen unzureichende Qualifizierung aufgezeigt und die Notwendigkeit der Qualitätssicherung propagiert. Einige Kommunen wie die Stadt Frankfurt haben inzwischen millionenschwere Qualitätssicherungsprogramme gestartet, doch offensichtlich ändert sich nichts Grundlegendes. Das konstatieren vor allem Verbände der älteren Menschen wie die Bundesarbeitsgemeinschaft der Seniorenorganisationen (Bagso 2002) oder das Deutsche Zentrum für Altersfragen (Adolph/ Heinemann 2003).

Nicht nur die Abhängigkeit der Versorgung von den finanziellen Mitteln der Pflegebedürftigen, sondern auch die lange Zeit fehlende Kontrolle der Leistungen hat dazu geführt, dass mit Altenheimen wahre Horrorszenarien assoziiert werden. Erst im Heimpersonalgesetz von 1993 (überarbeitet 1998) wurde festgelegt, dass HeimleiterInnen eine gewisse einschlägige Qualifikation benötigten. Die darin ebenfalls getroffene Regelung, dass mindestens $50 \%$ der in der Pflege Tätigen in einem Altenpflegeheim auch tatsächlich ausgebildete Pflegekräfte sein sollten, ließ sich gegen den erbitterten Widerstand der Träger bis heute nicht realisieren. Was denn überhaupt ausgebildete Altenpflegekräfte sind, darüber entschied jedes Bundesland, das gemäß den jeweiligen Landespflegegesetzen auch eine gewisse Kontrolle über die Heime hat, anders. Eine bundesweit einheitliche Altenpflegeausbildung mit einer Mindestdauer von drei Jahren gibt es erst seit dem letzten Jahr. Überhaupt findet erst seit der Einführung der Pflegeversicherung ein Mindestmaß an Kontrolle der Heimversorgung statt. Das am 1.2.2002 zum dritten Mal überarbeitete Heimgesetz soll den BewohnerInnen eine menschenwürdige Behandlung garantieren und zielt auf die Verschärfung der Heimaufsicht und -überprüfung. Diese Gesetzesänderung, die zunächst als "Patientenschutzgesetz ${ }^{\text {" }}$ in die Debatte eingeführt wurde (der Titel musste auf Grund der Proteste der Trägerverbände zurückgezogen werden, vgl. Pflege aktuell, 2000), verweist darauf, wie es in den Pflegeheimen tatsächlich aussieht.

Allerdings war auch die Einführung der Pflegeversicherung in keiner Weise dazu gedacht, den Personalbestand in den stationären Pflegeeinrichtungen auszubauen. Die Förderung der ambulanten und familiären Pflege zielte vielmehr zumindest implizit darauf, die vergleichsweise teure Heimunterbringung zu minimieren. Die Finanzierung der Heimunterbringung über die Pflegeversicherung sollte lediglich diejenige über die Sozialhilfe ersetzen, aber nicht über sie hinausgehen (Pabst 1999). Ein Dilemma, das neben den BewohnerInnen auch die ArbeitnehmerInnen betrifft: Die Heime sind personell unterbesetzt und die Beschäftigten sind schlecht bezahlt, denn ebenso wie die privaten Unternehmen entziehen sich vor allem kirchliche Träger den im öffentlichen Gesundheitswesen üblichen Tarifen. Physisch und psychisch überlastet, 
stehen die Beschäftigten den wachsenden Anforderungen ohnmächtig gegenüber. Gerade die weiblichen Migrantinnen unter ihnen sind meist noch stärker der Willkür der Arbeitgeber ausgeliefert. In Teilzeitbeschäftigungen mit geringen Qualifikationen ist es nahezu unmöglich, einen anderen Arbeitgeber zu finden oder sich entschieden für eine Verbesserung der Arbeitsbedingungen zu engagieren.

In diesem Bereich werden drei Dinge deutlich:

- Die Marktregulierung sorgt für eine verschärfte Abstufung der Leistungen je nach Einkommenslage bei gleichzeitiger verstärkter Ausbeutung der MitarbeiterInnen.

- Wenn Gesundheitsdienstleistungen nicht einer besonderen Kontrolle unterzogen werden, führt die Durchsetzung wirtschaftlicher Prinzipien keineswegs auch zu einer Verbesserung der Leistung.

- Die Beschäftigung von qualifizierten Vollzeitkräften wird auf ein absolutes Minimum beschränkt.

\subsection{Die ambulante Pflege}

Mit der Einführung der Pflegeversicherung boomte plötzlich der Markt der ambulanten Pflege. Existierten 1993 erst 6250 ambulante Dienste, von der Gemeindekrankenpflege bis zu mobilen sozialen Diensten, so konnten 1998 bereits 11.700 solcher Einrichtungen gezählt werden und 2000 rund 13.000 . Ungefähr die Hälfte davon wird von freigemeinnützigen Verbänden betrieben, $46 \%$ sind private Unternehmen und nur 5\% gehören einem öffentlichen Träger (GBE 1998: 316; Deutscher Bundestag 2001). Allerdings betreuen die privaten Anbieter durchschnittlich weniger PatientInnen als die anderen. Allein $20 \%$ der Privaten betreuen nicht mehr als ein bis zehn PatientInnen und nur 0,2\% haben einen Patientenstamm von über 150 PatientInnen. 1999 arbeiteten 184.000 Personen in ambulanten Einrichtungen, wovon $85 \%$ Frauen waren. $64 \%$ aller Beschäftigen arbeiteten in Teilzeit, der überwiegende Teil davon mit weniger als halben Stellen und als geringfügig Beschäftigte (Statistisches Bundesamt 2001: 11). Über die Qualifikationen der Beschäftigten liegen keine Zahlen vor. Doch allein aus der Tatsache, dass mehr als zwei Drittel aller Arbeitnehmerinnen in diesem Sektor in Teilzeit arbeiten, geringfügig beschäftigt sind oder Auszubildende und Zivildienstleistende sind, lassen sich Rückschlüsse auf die Arbeitsbedingungen ziehen.

Allerdings wirkte der Boom weniger beschäftigungswirksam als erwartet. Zwar hat sich die Zahl der in der ambulanten Pflege Beschäftigten verdreifacht, aber die erwartete Zahl von 400.000 neuen Arbeitsplätzen wurde bei weitem nicht erreicht. Diese wäre zu erwarten gewesen, wenn die Pflegebedürftigen überwiegend Sachleistungen und somit professionelle Pflege in Anspruch genommen hätten. Doch sie zogen mehrheitlich die Geldleistungen vor, über 
deren Ausgabe sie selbst verfügen können. Damit floss dieses Geld nicht in den formellen Arbeitsmarkt, sondern trug dazu bei, pflegende Angehörige, mehrheitlich Frauen, zur Pflege zu verpflichten und sich aus dem formellen Arbeitsmarkt zurückzuziehen (vgl. Pabst 1999). Andererseits, so ist zu vermuten, werden diese Geldleistungen auch genutzt, um Migrantinnen, d.h. vor allem Frauen aus Osteuropa, die jeweils für drei Monate mit einem Touristenvisum ins Land kommen, zu engagieren, die eine billige Versorgung anbieten können. Über das Ausmaß dieser Art von Beschäftigung gibt es kaum Zahlen, man muss jedoch davon ausgehen, dass es enorm ist. Nicht nur die letztjährige Legalisierung von osteuropäischen Pflegehilfen machte das offenbar. Mittlerweile gehen vor allem die freigemeinnützigen Anbieter (z.B. die AWO und das DRK) dazu über, Kurzkurse für Migrantinnen anzubieten, um ihnen grundlegende Kenntnisse zu vermitteln. Damit wird deutlich, dass diese Art der Pflegehilfe auf dem Wege ist, sich fest zu etablieren.

Mit der Frage der Beschäftigung von weiblichen Familienangehörigen und Migrantinnen in der Pflege ist erst ein Teil der stattfindenden Verschiebungen angesprochen. Insgesamt setzt die Politik in Bund und Ländern verstärkt auf die Einbezichung von Ehrenamtlichen in der Betreuung Pflegebedürftiger, wie die groß angelegten Kampagnen zur Aufwertung des Ehrenamts in den vergangenen Jahren zeigen (z.B. die Bundesregierung: „Förderung des bürgerschaftlichen Engagements"; oder Rheinland-Pfalz: „Das Ehrenamt - gut für alle“). Auch viele ProfessorInnen in den Pflegestudiengängen plädieren für eine Ausweitung der ehrenamtlichen Pflege und sehen eine der Hauptaufgaben der professionellen Pflege der Zukunft darin, die Laienpflege zu fördern und durch Beratung zu professionalisieren (Steppe 1992; Steinbock u.a. 2002).

Auch für die neu entstandenen Unternehmen zeigte sich schnell, dass die ambulante Pflege nicht wie anfänglich erwartet zur Goldgrube wurde. Die abrechenbaren Leistungen können offensichtlich nur dann profitabel erbracht werden, wenn sie nicht von Fachkräften ausgeführt werden. Stundensätze von z.B. 28,50 Euro in Hessen (FR 3.6.2003) und das bei minutengenauen Vorgaben für Einzelleistungen sind in der Tat nicht geeignet, irgendwelche Überschüsse zu erwirtschaften. Das heißt, dass offensichtlich nur das absolute Minimum an qualifzierten Pflegenden eingestellt und darüber hinaus mit Hilfen und Aushilfen gearbeitet wird. So stellte die Bundesregierung zur Unterrichtung des Bundestags bezüglich der Pflegeversicherung erhebliche Defizite in der Personalausstattung und der Qualifikation des vorhandenen Personals sowie die zunehmende Einstellung von ungelernten Kräften fest (Deutscher Bundestag 2001).

War die Situation für eine angemessene Qualitätssicherung von Anfang an insofern prekär, als sich entweder nur kleine ambulante Dienste, vielleicht besser als freiberufliche Krankenschwestern zu bezeichnen, unter rigoroser Selbstausbeutung oder einige wenige größere Unternehmen mit "geschickter" Personalpolitik durchsetzen konnten, so verschärft sich die Konkurrenzlage immer 
mehr. Seit die Krankenhäuser stärker in den ambulanten Sektor vordringen, bieten sie auch eigene ambulante Pflegedienste an. Da sie außerdem aufgrund ihrer Entlassungspolitik und mit der Möglichkeit des ambulanten Operierens sowie der steigenden ärztlichen ambulanten Versorgung aus den Krankenhäusern heraus einen unmittelbaren Zugang zu den PatientInnen haben, werden sie zu einer ernsthaften Konkurrenz für andere Unternehmen.

$\mathrm{Zu}$ den beschriebenen Tendenzen des Vordringens von semiprofessioneller bzw. Laienpflege und einer Umstrukturierung des Angebots kommen noch weitere Entwicklungen hinzu. Das sind der viel zitierte demografische Wandel, d.h. eine Zunahme des Anteils älterer und pflegebedürftiger Menschen, finanzielle Probleme in der Pflegeversicherung, ein prognostizierter Rückgang von Personen, die den Pflegeberuf ergreifen, und eine stabile hohe Arbeitslosigkeit. Alles zusammen spricht dafür, dass weite Teile der ambulanten pflegerischen Versorgung zukünftig vom informellen Arbeitsmarkt abgedeckt werden. ${ }^{5}$

\section{Perspektiven der Pflege}

Wie aus den Analysen der verschiedenen Sektoren des Gesundheitswesens deutlich wird, bewirkt die neoliberale Umgestaltung in den Arbeitsfeldern der beruflichen Pflege eine allmähliche Übertragung von Pflegetätigkeiten an Hilfskräfte, geringfügig Beschäftigte, wenn nicht gar an Laien. Hoch qualifizierte Pflegefachkräfte werden zukünftig wohl nur noch in einigen wenigen Teilbereichen gesucht werden. Eine solche hierarchische Ausdifferenzierung lässt sich an den Ausbildungskonzepten (Robert Bosch Stiftung 1992 u. 2000) und an den Tendenzen, die Grundausbildung (zumindest für einen Teil der Pflegenden) auf die Ebene der Fachhochschulen zu heben, ablesen. Diese Entwicklung wurde und wird von Teilen der Pflegeberufe mit vorangetrieben. Das geschieht zu einem wesentlichen Teil, um den Frauenberuf Pflege zu professionalisieren und aus der Unterordnung unter die MedizinerInnen zu lösen. An einer Relativierung ärztlicher Machtpositionen waren auch andere Akteure interessiert, so dass bei der Durchsetzung von Reformansätzen die Pflegeberufe in eine ambivalente Rolle gerieten.

Während alle Strukturreformen seit Mitte der 80er Jahre mehr oder weniger offen die Machtposition der Ärzteschaft anzukratzen versuchten, wurde die Pflege allem Anschein nach wesentlich gefördert. Mit der Reform des Krankenpflegegesetzes von 1985 wurde die Gleichwertigkeit der bundesdeutschen Pflegeausbildung mit der anderer EU-Länder gesichert. Das Gesundheitsreformgesetz von 1988 sorgte dafür, dass die Leistungen der häuslichen Krankenpflege erstmals von den Krankenkassen bezahlt wurden, wodurch es zu ei-

5 Eher zurückhaltende Prognosen über die Beschäftigungsentwicklung geben Schnacke (2002) und Wörz u.a. (2002), die jedoch nur vorsichtig auf die Möglichkeit des informellen Sektors verweisen. 
nem ersten Anstieg der Beschäftigung im Pflegebereich kam. Von 1991 an wurden ca. 40 Pflegestudiengänge eingerichtet, um die Qualität der Pflege zu verbessern, aber auch um den Beruf attraktiver zu machen (Mühlum u.a. 1997). Mit dem Gesundheitsstrukturgesetz von 1993 wurde ein neues Prinzip für die Berechnung der Pflegestellen in Krankenhäusern (Pflegepersonalregelung) eingeführt, das zu einem direkten Anstieg von Arbeitsplätzen führte. Die Einführung der Pflegeversicherung brachte einen weiteren Anstieg im stationären und ambulanten Pflegebereich. Seit 1995 ist die Pflege mit einer Stimme in der Konzertierten Aktion im Gesundheitswesen vertreten, und seit 1999 gibt es auch eine Vertreterin der Pflege im dazugehörigen Sachverständigenrat. Im Jahr 2000 richtete die damalige Bundesgesundheitsministerin Andrea Fischer eine WHO-Pflegekonferenz aus, bei der sich die GesundheitsministerInnen aller Länder der WHO-Region Europa zu unterstützenden Maßnahmen für die Weiterentwicklung der Pflege verpflichteten. Das gab den diversen pflegerischen Verbänden (konfessionelle, Rotes Kreuz, freie, Verbände der Lehrkräfte und der Pflegedienstleitungen u.v.a.m.) Anlass, sich der politischen Anerkennung als vollwertige Partner im Gesundheitswesen nahe zu fühlen.

Diese Veränderungen weckten nicht nur bei den Interessensverbänden, sondern auch bei der neuen Gruppe der PflegeakademikerInnen große Erwartungen bezüglich der Professionalisierung und beruflichen Autonomie der Pflege. Sie setzten auf die Qualifikation von Lehrenden für die Pflegeberufsausbildung und insbesondere auf ein verändertes Aufgabenprofil der leitenden Pflegekräfte, wie es die Robert Bosch Stiftung (1992) wegweisend definierte: Zum einen sollte die akademische Ausbildung leitende Pflegekräfte zu gleichberechtigten Partnern in der Patientenversorgung machen. Damit wurde implizit eine neue Machtverteilung angestrebt. Des weiteren sollten die Führungskräfte in der Pflege dazu befähigt werden, die Ökonomisierung in ihrem Bereich voranzutreiben bzw. Konzepte zu entwickeln, die pflegerische Notwendigkeiten mit ökonomischen Prinzipien verbinden sollten. Dass leitende Pflegekräfte eine wichtige Funktion bei der Umstrukturierung der Einrichtungen des Gesundheitswesens übernehmen könnten und sollten und dass aus diesem Grunde ein betriebswirtschaftlich ausgerichtetes Studium erforderlich sei, darüber bestand in der Literatur zur Akademisierung der Pflege weitgehender Konsens (vgl. Bock-Rosenthal 1999; Kraushaar, Müller 1993; Mühlum u.a.1997).

Für die immense Affinität mit den Zielen einer Politik, die Krankenhäuser und andere Einrichtungen in Wirtschaftsunternehmen umwandeln wollte, gibt es verschiedene Gründe: Da die Förderung der Pflege unmittelbar mit der Ökonomisierung des Gesundheitswesens einherging und die Pflege trotz der so genannten Kostendämpfungspolitik expandierte, fühlte sich die Pflegeelite eben diesen Prinzipien anscheinend besonders verbunden und erwartete eine weitere Aufwertung des Berufs gerade aufgrund der so genannten Krise. Zweitens ging es darum, die Position der Pflegedienstleitung, die in der berufsgeschicht- 
lichen Entwicklung immer als besondere Machtstellung galt (Schmidbaur 2002: 78-83), zu erhalten und zu modernisieren. Trotz erheblicher Einschränkungen, die die Bedeutung der Oberinnen und später Pflegedienstleitungen verringerten, bot diese Position doch bis in die 1990er Jahre der Pflege die Beteiligung an der Krankenhausleitung. Diese wurde durch die oben genannte Neuorganisation vom Dreiergremium zur Geschäftsführung in den Händen eines Managers bedroht. Durch die Verbesserung der Qualifikation der PflegemanagerInnen erhoffte man sich, weiterhin Einfluss auf die Krankenhausführung nehmen zu können, wenn nicht gar gleichberechtigt beteiligt zu werden.

Soweit die von der Berufsgruppe selbst vorgebrachten Enwartungen und Hoffnungen. Diese wurden vor allem darum von anderen unterstützt, weil die Krankenhäuser für die fundamentalen Umwälzungen modernes Managementpersonal benötigten, das die Akzeptanz der zu bewältigenden Reformen bei den Beschäftigten erhöhte. Wer wäre dazu besser geeignet gewesen als VertreterInnen der betroffenen Berufsgruppen, die sich zudem noch selbst dafür anboten. Während also die Pflegeeliten davon ausgingen, die Ökonomisierung für die Festigung und sogar Ausweitung der eigenen Position nutzen zu können, sahen die Krankenhausträger und wohl auch die Gesundheitspolitiker in der Pflege willige GehilfInnen bei der Umstrukturierung. Dies nicht zuletzt auch, weil die vorbehaltlose Mitwirkung der Pflege Auswirkungen auf die Zurückhaltung der Ärzteschaft versprach. Dass hier eine mögliche Instrumentalisierung des Pflegemanagements für die Durchsetzung neoliberaler Konzepte stattfand, wurde im pflegerischen Diskurs nicht thematisiert. In der entsprechenden Literatur zur Entwicklung von Pflege(management)studiengängen und zu den Aufgaben des mittleren und gehobenen Pflegemanagements kommen kritische Überlegungen nicht vor. Vielmehr wurden mit großem Enthusiasmus die Handlungspotenziale gepriesen, die sich aus den Veränderungen ergeben, so u.a.:

„Die Auswirkungen der Gesundheitsreformgesetzgebung schaffen Möglichkeiten zur betrieblichen Verselbständigung im gesamten Gesundheitssektor. Damit werden Handlungspotentiale der Pflegeberufe erweitert, die nur strategisch genutzt werden müssen, um die Qualität der Pflege zu verbessern, auf die Personalentwicklung und Organisationsentwicklung Einfluss zu nehmen und vor allem auch sich in die Unternehmensführung und das Management des Gesamtunternehmens einzuschalten." (Bock-Rosenthal 1999: 18)

„Infolge einer Zuspitzung von sozialem Pflegeproblem und Finanzierungsproblemen im deutschen Gesundheitswesen erlässt die zentralstaatliche Ebene gesetzliche Neuregelungen, die zu tiefgreifendem Wandel für die dezentrale Ebene der Pflegeorganisationen und Pflegeberufe führen. Durch die neue Pflegeversicherung sowie aktuelle Gesundheitsreformmaßnahmen verändern sich die Handlungsmuster von Pflegeorganisationen. Krankenhäuser, Altenheime und ambulante Pflegedienste wandeln sich von starren, passiv-ausführenden, bürokratischgewährleistenden, Anstalten' hin zu dynamischen, aktiv gestaltenden, nutzerorientierten, Sozial-Unternehmen". "(Landenberger 1998: 12)

Dementsprechend wurde von Verbänden, nicht zuletzt von der Bundesarbeitsgemeinschaft Leitender Krankenpflegepersonen, und Hochschulen gleichermaßen jedes neue Instrument der Ökonomisierung als pflegefördernd 
propagiert. Eifrig und kritiklos wurden Qualitätssicherung, Qualitätsmanagement, die Entwicklung von betriebs- und hauseigenen Pflege-, Philosophien " vorangetrieben, die Entwicklung von Pflegestandards in einzelnen Häusern, aber auch auf Bundesebene zum Prestigeobjekt erklärt. Bei der Einführung der DRGs beteiligten sich PflegemanagerInnen und Pflegeverbände, um Pflegetätigkeiten in Diagnoseschlüsseln zu fassen. Dabei war man vor allem bemüht die eigenen Leistungen sichtbar und berechenbar zu machen, und das obwohl die Erfahrungen der australischen Pflege mit demselben DRG-System klar und deutlich zeigten, dass mit dieser Berechnung vor allem in der Pflege eine Vielzahl von Stellen (18 - $20 \%$, siehe oben) entfallen würde. Die Frage, ob die Auswirkungen des eigenen Tuns für Krankenschwestern/-pfleger ohne besondere Zusatzqualifikationen oder für die PflegehelferInnen nicht bedacht oder einfach in Kauf genommen wurden, ist nicht leicht zu beantworten. Doch lassen die Intentionen, eine weitere Ausdifferenzierung innerhalb des Berufs (Robert Bosch Stiftung 2000) zu unterstützen, und die explizite Hoffnung darauf, dass die neue Elite an politischen und ökonomischen Entscheidungen beteiligt werden wird, den Schluss zu, dass letzteres der Fall ist.

In diesem Zusammenhang soll auch darauf hingewiesen werden, dass der legitimatorische Diskurs von der Selbstverantwortung in der Gesundheitspolitik (Kühn 1998) ebenfalls aufgenommen und als Konzept der Hilfe zur Selbstpflege reproduziert wurde. Somit wurde die Sorge um die wachsende Arbeitsbelastung im beruflichen Alltag nicht in beschäftigungspolitische Forderungen verwandelt, sondern in neoliberaler Ideologie aufgehoben.

Wie bereitwillig Leitungskräfte in der Pflege neue Ideen der Unternehmensführung aufnehmen, wird am Beispiel des Pflegedienstdirektors der Frankfurter Unikliniken deutlich. Er ist keineswegs ein Ausnahmefall, sondern eher typisch für die neue Generation von Pflegemanagern. Unter den beabsichtigten "Ausgründungen" des Uni-Klinikums wird es eine GmbH geben, die die Krankenpflegekräfte beschäftigt. Der Vorteil dabei liege für den Pflegedirektor in der Möglichkeit, aus dem BAT aussteigen und leistungsgerecht bezahlen zu können. Dazu können Arbeiten zwischen Fachkräften und Hilfskräften neu verteilt werden. Außerdem übernehme man dann auch die Überleitung vom Krankenhaus nach Hause zur Do-it-yourself-Betreuung, d.h. die Übertragung der Pflege an Laien (Lorenc 2003).

Letztendlich haben alle Bemühungen um mehr Autonomie, die die Pflegeelite sich als Ergebnis der Ökonomisierung des Gesundheitswesens versprach, wenig Erfolg gezeigt. Noch immer kann Pflege nur auf ärztliche Anordnung hin durchgeführt werden, können Pflegende nicht einmal Pflegehilfsmittel verschreiben. In der Leitung von Krankenhäusern bleiben sie immer weiter hinter Betriebswirten zurück, für die es in den letzten Jahren zunehmend neue Qualifizierungen für Leitungsfunktionen im Gesundheitswesen gibt. In allen Einrichtungen des Gesundheitswesens werden Pflegepersonalmangel, erhöhter 
Arbeitsdruck und der Einsatz von nicht ausreichend qualifiziertem Hilfspersonal beklagt. Die Erwartungen, dass der demografische Wandel und die Zunahme von chronischen Erkrankungen zu mehr Pflegebedürftigkeit führen, trafen zwar ein, nur leitete sich daraus nicht $a b$, dass dieser Bedarf durch eine adäquat expandierende pflegerische Versorgung gedeckt wird.

\section{Allgemeine Tendenzen}

Abschließend sollen noch einmal die grundlegenden Tendenzen und deren Auswirkungen für die Beschäftigten im Gesundheitswesen skizziert werden. Die immer schneller voranschreitende Ökonomisierung des Gesundheitswesens hat neben einer expandierenden Privatisierung auch der allgemeinen Gesundheitsversorgung wesentliche Veränderungen zur Folge. Hoch qualifiziertes Gesundheitspersonal wird es voraussichtlich nur noch in wenigen zentralen Bereichen geben, während viele Leistungen von billigeren Arbeitskräften übernommen werden. Diese Entwicklung hat bereits der Sachverständigenrat im Gesundheitswesen im Gutachten 2000/2001 angesprochen, als er darauf hinwies, dass in der Alten- und ambulanten Pflege zwischen 1996 und 1999 der Anteil der Fachkräfte zurückging, während die Zahl der Mitarbeiter ohne Qualifikation um das sechsfache gestiegen war (Sachverständigenrat 2002: 105).

Darüber hinaus werden einerseits technische, hauswirtschaftliche etc. Arbeitsbereiche aus den Gesundheitseinrichtungen ausgegliedert und als Zulieferfirmen ausgegründet oder an solche vergeben. Damit wird ein großer Teil des Personals vom öffentlichen in den privaten Dienstleistungssektor entlassen. Auf der anderen Seite entstehen große Mischkonzerne, wie wir sie heute bereits von den börsennotierten Klinikbetreibern kennen, die vom Krankenhaus der Allgemeinversorgung bis zur Rehabilitationseinrichtung mit zugehörigen Wellnessangeboten oder von der Seniorenwohnanlage bis zum Pflegeheim alles bieten, also auch Hotel- und Freizeitangebote im Programm haben. Die Folgen für die ArbeitnehmerInnen und die Arbeitsverhältnisse lassen sich leicht ablesen aus den bekannten Entwicklungen im Hotelgewerbe bzw. aus Fitnesseinrichtungen - von geringer Bezahlung über schlechte Arbeitsbedingungen bis hin zu ungeregelten Arbeitszeiten.

Mit diesen Entwicklungen liegt das Gesundheitswesen ganz im Trend der Zeit, wenn es nicht sogar als einer der wichtigsten Wirtschaftsbereiche zum Trendsetter wird. Auf jeden Fall entsprechen die Verschiebungen der Beschäftigung im Gesundheitswesen den Prognosen für den gesamten Arbeitsmarkt, die ein Ansteigen der Frauenbeschäftigung von 42,2\% 2000 auf 44,6\% im Jahr 2010 voraussagen. Allerdings wird es sich dabei vor allem um Verschiebungen von Vollzeitarbeitsplätzen auf Teilzeitarbeitsplätze handeln, also um eine Umverteilung in der Gruppe der Frauen (Engelbrech/Jungkunst 2001: 318).

Diese Entwicklungen schreiten seit einem Jahrzehnt voran und werden im Fal- 
le der (Teil-)Privatisierung von Krankenhäusern der Allgemeinversorgung von lokalen Protesten von Beschäftigten und Gewerkschaften, zum Teil auch von BürgerInnen oder kommunalen Oppositionsparteien begleitet. Insgesamt jedoch geht die Umstrukturierung der Arbeitsverhältnisse und -bedingungen relativ reibungslos voran. Das ist sicherlich der Tatsache geschuldet, dass es sich dabei um einen Prozess in kleinen Schritten handelt, von dem immer nur einzelne Einrichtungen oder Berufsgruppen betroffen sind. Zum anderen dürfte das jedoch auch darauf zurückzuführen sein, dass unser Gesundheitswesen nie ein staatliches war und somit ein großer Teil der Einrichtungen in der Hand von freigemeinnützigen Trägern, darunter den Kirchen war. Für letztere gelten die von ÖTV und DAG bzw. ver.di ausgehandelten Mantelund Entgelttarifverträge nicht; der BAT galt also schon immer nur für einen Teil der Beschäftigten. Des Weiteren gab es im Pflegebereich traditionell eine besondere Art der Anstellung, die auch heute noch vorkommt. Freigemeinnützige Verbände schlossen mit Krankenhäusern Verträge über die Gestellung von Pflegepersonal, d.h. die Pflegekräfte waren bei den Verbänden angestellt und wurden an das Krankenhaus ausgeliehen. Auch damit geht eine besondere Art des Beschäftigungsverhältnisses einher, die wohl nur der modernen Leiharbeit vergleichbar ist. Die neuen Entwicklungen schließen also teilweise übergangslos an alte Zustände an; damit erklärt sich, warum diese Umstrukturierungen relativ reibungslos durchgeführt werden können.

\section{Literatur}

Adolph, Holger; Heinemann, Heike (2003): DZA-Expertenworkshops zur Pflegequalität - Teil 1. Die Zukunft der Personalsituation in der Altenpflege gestalten. informationsdienst altersfragen (Berlin), 30. Jg., 2/2003, S. 2-5.

Ahsen, H. B. von; Grashoff, Dietrich (2003): Der Zusammenschluss kommunaler Krankenhäuser in einer gemeinnützigen GmbH. Das Krankenhaus (Düsseldorf) 5, S. 369-374.

BAGSO Nachrichten online, 4/2002 www.bagso.de/888/04_02_07.htm

Bock-Rosenthal, Erika (1999): Von der Krankenschwester zur Managerin. Akademisierung oder Professionalisierung. In: dies. (Hrsg.): Professionalisierung zwischen Praxis und Politik. Der Modellstudiengang Pflegemanagement an der Fachhochschule Münster. Bern: Hans Huber S. 1741.

Care konkret (2003): Das aktuelle CAREkonkret-Interview mit Prof. Großkopf: Kündigungswelle "dank" DRGs? Care Konkret, 23.3.2003, Hannover: Vincentz Verlag

Bandelow, Nils C. Gesundheitspoltitik (1998): Der Stat in der Hand einzelner Interessengruppen? Opladen: Leske + Budrich.

Deppe, Hans-Ulrich (2000): Zur sozialen Anatomie des Gesundheitssystems. Neoliberalismus und Gesundheitspolitik in Deutschland. Frankfurt/M.: VAS.

Deutscher Bundestag, 14. Wahlperiode (2001): Unterrichtung der Bundesregienng. Zweiter Bericht über die Entwicklung der Pflegeversicherung. Drucksachen des Deutschen Bundestags $14 / 5590$, Berlin.

Engelbrech, Gerhard; Jungkunz, Maria (2001): Arbeitsmarktperspektiven für Frauen bis 2010. WSI Mitteilungen 54, 5/2001, S. 317-322.

GBE (1998): Statistisches Bundesamt (Hrsg.) (1998): Gesundheitsbericht für Deutschland. Stuttgart: Metzler-Poeschl.

Heimstatistik (2003): Alten- und Behinderteneinnchtungen. Deutsches Zentrum für Altersfragen, Berlin. Henniger, Wolfgang (2003): Von Altenpflege bis Wellness. Das Gesundheitswesen ist einer der 
Megamärkte von morgen - auch für die Berufsausbildung. Frankfurter Rundschau, 15.2.2003, Beilage Berufsrundschau, S. 1

Informationssystem der Gesundheitsberichterstattung des Bundes. www.gbe-bund.de

Kraushaar, D.; Müller, M.: Der 'Studiengang Pflege' an der Fachhochschule Frankfurt am Main. In: Fietzek, L.; Kraushaar, D. (Hrsg.): Fachhochschule Frankfurt. Pflege auf dem Weg zur Hochschule. Frankfurt/M.: Fachhochschulverlag 1993.

Kühn, Hagen (1998): „Selbstverantwortung“ in der Gesundheitspolitik. In: Jahrbuch für kritische Medizin. Berlin: Argument-Verlag. S. 7-20.

Landenberger, Margarete (1998): Innovatoren des Gesundheitssystems. Dezentrale Handlungspotentiale von Pflegeorganisationen und Pflegeberufen durch die neue Gesetzgebung. Bern: Hans Huber.

Lorenc, Anne (2003): „Handeln wie ein Wirtschaftskonzern". Uniklinikum will mehr Kooperation und Transparenz. Frankfurter Rundschau. Stadtausgabe, 12.2.2003. S. 23

Michelsen, Kai (2002): Blockade oder Stabilisierungsfaktor? Die Selbstverwaltung in der Kritik. In: Deppe, Hans-Ulrich u.a. (Hrsg.) Solidarische Gesundheitspolitik. Hamburg: VSA, S. 104-115.

Molitor, Martin; Walger, Martin (2003): Bereitschaftsdienst und Arbeitszeit: Der aktuelle Stand der rechtlichen und politischen Diskussion. Das Krankenhaus (Düsseldorf) 5, S. 363-366.

Morone, James A. (1996): Politik, Märkte und Gesundheitsreform. Amerikanische Gesundheitsvorsorge für Deutschland? In: Behrens, Johann u.a. (Hrsg.): Gesundheitssystementwicklung in den USA und Deutschland. Baden-Baden: Nomons, S. 49-63.

Mühlum, Albert; Bartholomeyczik, Sabine; Göpel, Eberhard (1997): Sozialarbeitswissenschaft, Pflegewissenschaft, Gesundheitswissenschaft. Freiburg: Lambertus.

Norddeutsche Landesbank (2002): Global Markets. Krankenhausmarkt im Umbruch. www.nordlb.com

Pabst, Stefan (1999): Mehr Arbeitsplätze für Geringqualifizierte nach Einführung der Pflegeversicherung? WSI-Mitteilungen 4, S. 234-240.

Robert Bosch Stiftung (1992): Pflege braucht Eliten. Denkschrift zur Hochschulausbildung für Lehr- und Leitungskräfte in der Pflege. Gerlingen: Bleicher Verlag.

Robert Bosch Stiftung (2000): Pflege neu denken. Zur Zukunft der Pflegeausbildung. Stuttgart: Schattauer.

Sachverständigenrat für die Konzertierte Aktion im Gesundheitswesen (2002): Gutachten 2000/2001. Bedarfsgerechtigkeit und Wirtschaftlichkeit. Bd. II. Baden-Baden: Nomos.

Schmacke, Norbert (2002): Förderung der Gesundheit, Nutzung des medizinischen Fortschritts und Förderung des Wirtschaftswachstums: Quadratur des Kreises. Arbeit und Sozialpolitik, 56. Jg., 5-6/2002. S. 10-15.

Schmidbaur, Marianne (2002): Vom "Lazaruskreuz“ zu "Pflege aktuell“. Professionalisierungsdiskurse in der deutschen Krankenpflege 1903 - 2000. Königstein: Ulrike Helmer Verlag.

Statistisches Bundesamt (2001): Kurzbericht: Pflegestatistik 1999. Pflege im Rahmen der Pflegeversicherung. Bonn.

Steppe, Hilde (1993): Perspektiven der professionellen Pflege - Krankenpflege im Dienst der Gesundheit. In: Müller, Hans-Werner (Hrsg.): Pflegenotstand Not der Pflegenden und Gepflegten. Krankenpflege im Dienst der Gesundheit; Kongressbericht 1992. Frankfurt/M.: Deutsche Zentrale für Gesundheitspflege, S. 115-128.

Steinbock, S.; Zegelin-Abt, A.; Büker, Ch. (2002): Pflegeberatung zu Hause etablieren. Forum Sozialstation, 26. Jg., 116, Mai. S. 36-38.

Stone, Deborah, A.: Das strategische Verhalten der Versicheter unter der Marktreform. Die politischen und kulturellen Auswirkungen von Wettbewerb. In: Behrens, Johann u.a. (Hrsg.): Gesundheitssystementwicklung in den USA und Deutschland. Baden-Baden: Nomos 1996. S. 35-48.

ver.di (2002): Infodienst Krankenhäuser, 18, 11/2002.

Wanek, Volker (1994): Machtverteilung im Gesundheitswesen. Struktur und Auswirkungen. Frankfurt: VAS.

Wismar, Matthias (1996): Gesundheitswesen im Übergang zum Postfordismsus. Die gesundheitspolitische Regulierung der Fordismuskrise in Großbritannien und der Bundesrepublik Deutschland. Frankfurt/M.: VAS.

Wohlfahrtsliga erhebt Klage gegen Krankenkassen. Frankfurter Rundschau, Stadtausgabe, 13. Juni 2003, S. 29.

Wörz, Markus u.a. (2002): Gesundheitswesen und Arbeitsmarkt: Das Beispiel NordrheinWestfalen. Sozialer Fortschritt 51. Jg., 7-8/2002. S. 183-188. 